\title{
Pinning and phonon localization in Frenkel-Kontorova models on quasiperiodic substrates
}

\author{
T. S. van Erp and A. Fasolino \\ Research Institute for Materials, Institute of Theoretical Physics, University of Nijmegen, Toernooiveld, NL-6525ED, \\ Nijmegen, The Netherlands \\ O. Radulescu \\ IRC in Polymer Science and Technology, Department of Physics, University of Leeds, Leeds LS2 9JT, United Kingdom \\ T. Janssen \\ Research Institute for Materials, Institute of Theoretical Physics, University of Nijmegen, Toernooiveld, NL-6525ED, \\ Nijmegen, The Netherlands \\ (Received 12 February 1999)
}

\begin{abstract}
We show that the static and dynamic properties of the Frenkel-Kontorova (FK) model drastically change when an incommensurate harmonic is added to the periodic potential. Our model consists of a harmonic chain with spacing $l$ on a quasiperiodic substrate potential of the form $V(x)=\sigma K /(2 \pi)^{2}[1-\cos (2 \pi x)]$ $+(1-\sigma) K /(2 \pi \tau)^{2}[1-\cos (2 \pi \tau x)]$, where the three relevant lengths $l, 1$, and $\tau^{-1}$ are chosen to be mutually incommensurate. Within this model we identify two classes of behavior. One presents a sliding mode up to an analyticity breaking, as in the FK model, and another is pinned for any strength of the additional harmonic. Besides, we show that in all cases if $\sigma \neq 0$ or 1, localization of phonons exists beyond a critical value of the potential strength. [S0163-1829(99)12629-8]
\end{abstract}

\section{INTRODUCTION}

The prototype model to describe systems where competition between two incommensurate lengths determines the ground-state energy is the Frenkel-Kontorova (FK) (Refs. 1 and 2) model which describes a harmonic chain with spacing $l$ subjected to a periodic incommensurate substrate potential. A remarkable feature of the model is the so-called analyticity breaking transition pointed out by Aubry ${ }^{2-4}$ where a structural transition with concomitant disappearance of a zerofrequency sliding mode occurs at a critical value $K_{c}$ of the strength of the external potential. This model has been used to study a variety of quasi-one-dimensional physical phenomena such as charge-density waves (CDW), ${ }^{5,6}$ adsorbate layers on surfaces, ${ }^{7}$ misfit dislocations, ${ }^{1,8}$ modulated structures, ${ }^{9}$ and composite structures. ${ }^{10,11}$ A new reason of interest is given by the recent finding that incommensurability between lattice parameter and substrate potential may lead to a regime of vanishing friction ${ }^{12-14}$ for which experimental evidence has been found. ${ }^{15}$

In this paper, we study both the ground state and the phonons of a generalized FK model with a quasiperiodic (QP) substrate potential. QP potentials represent an intermediate situation between periodic order and disorder. The lack of periodicity allows states which are extended, localized, or neither of the two. A study of the nature of excitations in QP structures can therefore contribute to the still open problem of determining the necessary and sufficient conditions for localization in incommensurate structures. ${ }^{4,16,17}$

The FK model is the limiting case of our QP potential and will be used as a reference system in the presentation of our results. Quasiperiodic potentials bring in one qualitatively new feature beyond the obvious one of breaking the translational invariance of the substrate, namely the height of the potential maxima becomes space dependent. We will show that in this situation, depending on the involved lengths, the analyticity breaking transition can either occur or be absent and that a second transition takes place at larger values of the potential strength. We also observe a transition to localized states in the phonon spectrum and make conjectures concerning the reasons for its occurrence in QP potentials and why localization is suppressed in the FK model.

The paper is organized as follows: in Sec. II we introduce our model, in Sec. II A we briefly review some basic properties of the FK model which are of relevance for the following, in Sec. II B we examine the conditions for the existence of a sliding mode for QP potentials by introducing what we call the "density criterion." This criterion helps in understanding the known properties of the FK model as well. In this subsection we also identify, within our model, two classes of systems displaying rather different behavior of the ground-state and phonon spectra. One presents a sliding mode up to an analyticity breaking, as in the FK model, in the other the mode is pinned for any strength of the additional harmonic. In Sec. III we begin the discussion of the numerical results by describing the numerical implementation of the model. Results for the ground-state and phonon spectrum are presented in Sec. III A, while phonon localization is dealt with in Sec. III B. Finally, in Sec. IV we summarize our findings and give conclusions and perspectives of this work.

\section{MODEL}

We consider a system described by a total potential energy which involves three lengths, $1, l$, and $\tau^{-1}$, which we take to be mutually incommensurate: 


$$
\begin{aligned}
E=\sum_{i=1}^{N}[ & \left.\frac{1}{2}\left(x_{i+1}-x_{i}-l\right)^{2}+V^{Q P}\left(x_{i}\right)\right], \\
V^{Q P}(x)= & \frac{K \sigma}{(2 \pi)^{2}}[1-\cos (2 \pi x)] \\
& +\frac{K(1-\sigma)}{(2 \pi \tau)^{2}}[1-\cos (2 \pi \tau x)] .
\end{aligned}
$$

The potential $V^{Q P}(x)$ represents a QP potential for $\tau$ an irrational number if $\sigma \neq 0$ or 1 . The parameter $K$ is the potential strength and $\sigma$ determines the relative ratio of the two harmonics. All energies are scaled by the elastic spring constant.

\section{A. Sliding mode in the FK model}

The original FK model represents the limiting case for $\sigma=0$ or 1 . The FK ground-state configuration $x_{i}^{G}$ for $K=0$ is given by equidistant positions $x_{i}=i l+\alpha$, where $\alpha$ is an arbitrary phase. For $K \neq 0$ the ground state can be described ${ }^{3}$ by a hull function $f(x)$ as $x_{i}^{G}=f(i l+\alpha)=i l+\alpha+g(i l$ $+\alpha$ ), where $g$ is often called the modulation function. The modulation function $g(x)$ in first order in $K$ (Ref. 13) reads

$$
g(x)=\frac{-K}{4 \pi} \frac{\sin (2 \pi x)}{1-\cos (2 \pi l)}+O\left(K^{2}\right)
$$

For the FK model it is well known ${ }^{3}$ that, for $N \rightarrow \infty$, if $l$ is incommensurate with respect to the period of the potential, the equations of motion for the displacement from equilibrium $\epsilon_{i}=x_{i}-x_{i}^{G} \sim e^{i \omega t}$ :

$$
\left[2-\omega^{2}+V^{\prime \prime}\left(x_{i}^{G}\right)\right] \epsilon_{i}-\epsilon_{i-1}-\epsilon_{i+1}=0
$$

have a solution $\omega^{2}=0$ for $K<K_{c}$ with eigenvector $\epsilon_{i}$ $=f^{\prime}(i l+\alpha)$, where $f^{\prime}$ is the derivative of the hull function. This mode is called sliding mode because it corresponds to a nonrigid displacement of the chain with respect to the substrate. One can describe it as a modulated acoustical mode. In fact, we can rewrite $\epsilon_{i}=1+g^{\prime}(i l+\alpha)$, i.e., a constant plus a modulation. This, in turn, means that for $K<K_{c}$ there is a continuum of ground states and that the chain can continuously slide with no energy cost from one to the other. Above $K_{c}$ the hull function becomes discontinuous, the manifold of ground states becomes discrete, the sliding mode gets a nonzero frequency, and the chain becomes pinned. The lowest phonon frequency is often called the phonon gap.

\section{B. Conditions for the existence of a sliding mode in QP potentials}

In the QP case three lengths play a role, $l, 1$, and $\tau^{-1}$. In the Fourier spectrum they give $l^{-1}, 1$, and $\tau$. We have to distinguish different cases according to the rank of the module generated by these Fourier vectors. If the equation

$$
n+m l+p l \tau=0, \quad n, m, p \text { integers, }
$$

has no trivial solution the rank is three, otherwise it is less. We have found that in the QP case $(\sigma \neq 0$ or 1$)$ a sliding mode occurs only if the rank is three, and then it exists up to a critical value of $K$. If the rank is less the sliding mode disappears for any value of $K$ if $\sigma \neq 0$ or 1 . Both cases can occur for different choices of the three relevant, mutually incommensurate, lengths. Below we will define the prototype cases I and II as examples of the two situations.

The condition (5) can be better understood in analogy to the FK model, where the incommensurability of the lattice parameter $l$ to the potential period means that the equidistant lattice positions are uniformly distributed over all potential values, since the quantities $X_{i} \equiv i l \bmod (1)$ for $i=$ $-\infty, \ldots, \infty$ form a dense set in the interval $[0,1]$, the unit cell of the periodic potential. In this case the sliding mode exists up to a critical potential strength. If the chain is commensurate, the distribution is not dense, implying that not all potential values are probed. In this case the chain is pinned for any $K \neq 0$ and the sliding mode is absent.

A QP potential of the form (1) can also be expressed as a periodic potential albeit in a two-dimensional (2D) space, namely

$$
\begin{aligned}
\widetilde{V}(x, y)= & \frac{K \sigma}{(2 \pi)^{2}}[1-\cos (2 \pi x)] \\
& +\frac{K(1-\sigma)}{(2 \pi \tau)^{2}}[1-\cos (2 \pi y)]
\end{aligned}
$$

out of which, the original potential $V^{Q P}(x)$ is found as intersection with the line of slope $\tau, V(x)=\widetilde{V}(x, \tau x)$. The condition for the existence of a sliding mode requires, as in the FK model, the undistorted configuration to be dense in the 2D unit cell of the periodic potential $\widetilde{V}(x, y)$ namely

$$
\left(X_{i}, Y_{i}\right) \equiv(i l, i \tau l) \bmod \mathbb{Z}^{2} \text { for } i=1 \cdots N,
$$

for $N \rightarrow \infty$ is a dense set in the unit cell of $\mathbb{Z}^{2}$.

We consider the following two exemplary cases:

(I) $l=\tau=\tau_{g}$, with $\tau_{g}=(\sqrt{5}-1) / 2 \sim 0.618$ the golden mean satisfying $\tau_{g}^{2}+\tau_{g}-1=0$.

(II) $l=\tau=\tau_{s}$, with $\tau_{s} \sim 0.755$ the spiral mean ${ }^{18}$ satisfying $\tau_{s}^{3}+\tau_{s}^{2}-1=0$.

Case I does not satisfy the above criterion of being densely distributed in the 2D unit cell. In fact:

$$
\begin{aligned}
\left(X_{i}, Y_{i}\right) & \equiv(i l, i l \tau) \bmod \mathbb{Z}^{2} \\
& =\left(i \tau_{g}, i \tau_{g}^{2}\right) \bmod \mathbb{Z}^{2} \\
& =\left[i \tau_{g}, i\left(1-\tau_{g}\right)\right] \bmod \mathbb{Z}^{2}=\left(i \tau_{g},-i \tau_{g}\right) \bmod \mathbb{Z}^{2}
\end{aligned}
$$

with all points folded back to a line $Y=1-X$. For vanishing $K$ this line can be shifted freely in the 2D unit cell but as soon as $K \neq 0$ it gets pinned at one particular line $Y=Y_{0}$ $-X$ with $Y_{0}=1 / 2$ in this case. Physically this implies a pinning of the chain in the most favorable energetic position corresponding to a nondegenerate ground state. Conversely, for case II the points are uniformly distributed in the 2D unit cell and the chain can move continuously from ground state to ground state with no energy cost as in the original FK model.

We show now how the density condition can be corroborated by a calculation of the lowest phonon frequency up to 
second order in $K$. By summing over $i$ [Eq. (4)] and applying periodic boundary conditions we find

$$
\sum_{i=1}^{N}\left[V^{\prime \prime}(i l+\alpha+g(i l+\alpha))-\omega^{2}\right] \epsilon_{i}=0
$$

which yields $\omega^{2}=0$ if we take $\epsilon_{i}=1+O(K)$ in first order in $K$. Going over to second order in $K$ we can generalize the expression (3) to that resulting from $\mathrm{V}^{Q P}$ as

$$
\begin{aligned}
g(x)= & \frac{-K \sigma}{4 \pi} \frac{\sin (2 \pi x)}{1-\cos (2 \pi l)} \\
& -\frac{K(1-\sigma)}{4 \pi \tau} \frac{\sin (2 \pi \tau x)}{1-\cos (2 \pi \tau l)}+O\left(K^{2}\right)
\end{aligned}
$$

and its two-dimensional periodic extension:

$$
\begin{aligned}
\tilde{g}(x, y)= & \frac{-K \sigma}{4 \pi} \frac{\sin (2 \pi x)}{1-\cos (2 \pi l)}-\frac{K(1-\sigma)}{4 \pi \tau} \frac{\sin (2 \pi y)}{1-\cos (2 \pi \tau l)} \\
& +O\left(K^{2}\right) .
\end{aligned}
$$

By expanding $V^{\prime \prime}$ as

$$
\begin{aligned}
V^{\prime \prime}(i l+\alpha+g(i l+\alpha))= & V^{\prime \prime}(i l+\alpha) \\
& +V^{\prime \prime \prime}(i l+\alpha) g(i l+\alpha)+O\left(K^{3}\right)
\end{aligned}
$$

and by using $\epsilon_{i}=1+g^{\prime}(i l+\alpha)+O\left(K^{2}\right)$ with the first-order expression of $g$ given by Eq. (9) into Eq. (8) we get

$$
\begin{aligned}
N \omega^{2}= & \sum_{i=1}^{N}\left[V^{\prime \prime}(i l+\alpha) g(i l+\alpha)\right. \\
& \left.+V^{\prime \prime \prime}(i l+\alpha) g(i l+\alpha)\right] .
\end{aligned}
$$

Notice that the above expression is valid with the first-order expansion of $g(x)$ because $\omega^{2}$ is zero in first-order approximation. The above sum can be transformed into an integral in the $2 \mathrm{D}$ unit cell of the periodic potential if the points are uniformly distributed in it as in case II or onto a line integral if the points fall onto a line as in case I. It is then straightforward to show that it yields $\omega^{2}=0$ in the first case, while in the case I integration over the line $Y=1 / 2-X$ leads to

$$
\begin{aligned}
\omega^{2}= & \int_{0}^{1}\left[\widetilde{V}^{\prime \prime}\left(X, \frac{1}{2}-X\right) \tilde{g}^{\prime}\left(X, \frac{1}{2}-X\right)\right. \\
& \left.+\widetilde{V}^{\prime \prime \prime}\left(X, \frac{1}{2}-X\right) \tilde{g}\left(X, \frac{1}{2}-X\right)\right] d X
\end{aligned}
$$

yielding

$$
\omega^{2}=\frac{3+2 \tau_{g}}{4} \frac{\sigma(1-\sigma)}{1-\cos \left(2 \pi \tau_{g}\right)} K^{2}+O\left(K^{3}\right)
$$

which is zero only for $\sigma=0,1$ as we wanted to prove. Formula 17 reproduces very well the numerical results of Fig. 1 for small $K$.

\section{NUMERICAL RESULTS}

Our numerical study begins by finding the ground-state structure for given values of the parameters. In order to simulate an infinite number of particles and get rid of surface effects, we use periodic boundary conditions $V^{Q P}(x)$ $=V^{Q P}(x+N l)$. This means that we have to choose commensurate approximations for $l$ and $\tau$ so that the model is placed on a torus of length $l \times N=P \times 1=R \times \tau^{-1}$ with $R$ and $P$ integers. It is well known that the golden mean $\tau_{g}$ can be approximated by ratios of the Fibonacci numbers $\left(F_{1}\right.$ $=1, F_{2}=1, F_{n}=F_{n-1}+F_{n-2}$ for $n>2$ ), namely

$$
\tau_{g}=\lim _{n \rightarrow \infty} \frac{F_{n}}{F_{n+1}} .
$$

Similarly the spiral mean can be approximated by ${ }^{18}$

$$
\tau_{s}=\lim _{n \rightarrow \infty} \frac{G_{n}}{G_{n+1}}
$$

with $G_{n+1}=G_{n-1}+G_{n-2}$ and $G_{-2}=G_{0}=1, G_{-1}=0$. Therefore we choose for case I

$$
N=F_{n+1}, \quad l=\frac{F_{n}}{F_{n+1}}, \quad \tau=\frac{F_{n-1}}{F_{n}}
$$

and for case II

$$
N=G_{n+1}, \quad l=\frac{G_{n}}{G_{n+1}}, \quad \tau=\frac{G_{n-1}}{G_{n}} .
$$

Note that periodic boundary conditions require us to use two different approximations for $l$ and $\tau$ although we should like to have them both equal to the golden or spiral mean.

\section{A. Ground state and phonons of QP systems}

In the FK model the Aubry transition is characterized by the discontinuity of the hull function and concomitant opening of a phonon gap at the critical value of the potential strength $K_{c}$. Another quantity of interest is the participation ratio (PR) which can describe the extended or localized character of the phonon modes. It is defined as

$$
\mathrm{PR}=\frac{1}{N} \frac{\left(\sum_{i}^{N} \epsilon_{i}^{2}\right)^{2}}{\sum_{i}^{N} \epsilon_{i}^{4}}
$$

and it is equal to 1 for extended modes and equal to zero for completely localized ones. In Fig. 1 we compare the dependence of these quantities on the potential strength $K$ for the FK model with those for the QP cases I and II with $\sigma$ $=0.25$. This figure summarizes our results and will form the base for the following discussion which will require reference to all other figures before the presentation of the results contained in it can be completed.

As expected on the basis of the density criterion, the phonon gap in case I is nonzero for any $K>0$, as opposed to the FK model and to the QP case II where it is zero up to $K_{c}$ 


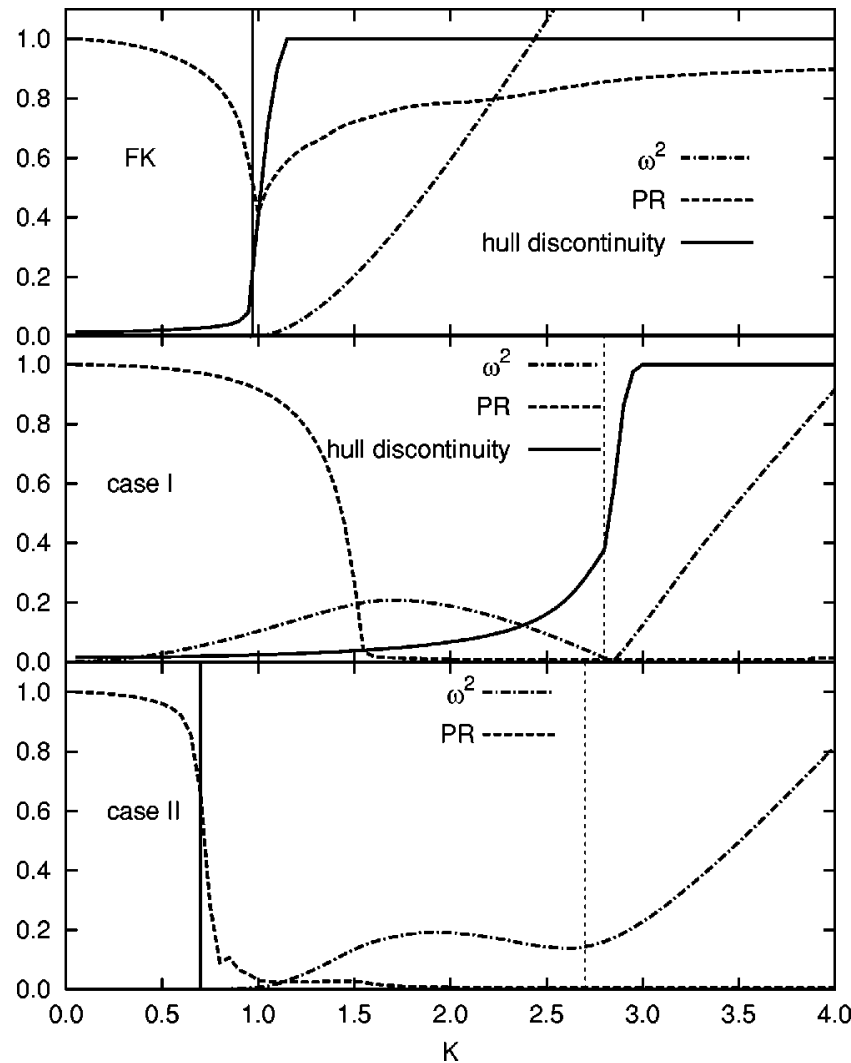

FIG. 1. Phonon gap (dash-dotted line), normalized largest discontinuity in the hull function (solid line), and participation ratio (PR) (dashed line) versus $K$ for the following: Top panel: FK model, $N=233, l=144 / 233 \approx \tau_{g}$, and $\sigma=1$. Results for the case $\sigma=0$ coincide with these. The solid vertical line indicates $K_{c}$. Middle panel: quasiperiodic case I (see Sec. II B), $N=233, l$ $=144 / 233, \tau=89 / 144$, and $\sigma=0.25$. The dashed vertical line indicates $K_{c}^{\prime}$. Bottom panel: quasiperiodic case II (see Sec. II B), $N$ $=351, l=265 / 351, \tau=200 / 265$, and $\sigma=0.25$. The solid vertical line indicates $K_{c}$, the dashed vertical line indicates $K_{c}^{\prime}$.

$\sim 1$ and $K_{c} \sim 0.7$ (for $\sigma=0.25$ ), respectively. ${ }^{19}$ The numerical results for $\omega^{2}$ in case I are very well reproduced for low $K$ by formula (17).

In Figs. 2 and 3 we show the 2D periodic unit cell of $\widetilde{V}(x, y)$ with all positions $x_{i}^{G}$ folded into it as $\left(x_{i}^{G}, \tau x_{i}^{G}\right) \bmod$ $\mathbb{Z}^{2}$ for increasing values of $K$. These figures give an image of the structural behavior of the ground state as a function of $K$. It can be clearly seen that for case I all points fall onto a line which first deforms and eventually becomes discontinuous, while in case II the points are very uniformly distributed up to the critical value $K_{c} \sim 0.7$, where the phonon gap opens up.

Coming back to Fig. 1, another interesting feature, common to both QP cases is the nonmonotonic dependence of the lowest phonon frequency on $K$. There is in both cases a minimum after which $\omega^{2}$ grows almost linearly with $K$. The occurrence of the minimum signals in both cases I and II the fact that no particles are anymore situated on maxima of the potential. We will come back to this point while discussing Figs. 4 and 5. Here we just want to point out that the low values of $\omega^{2}$ for a large range of $K$ may be of importance for the friction force and the depinning transition. We plan to study this aspect in the future.

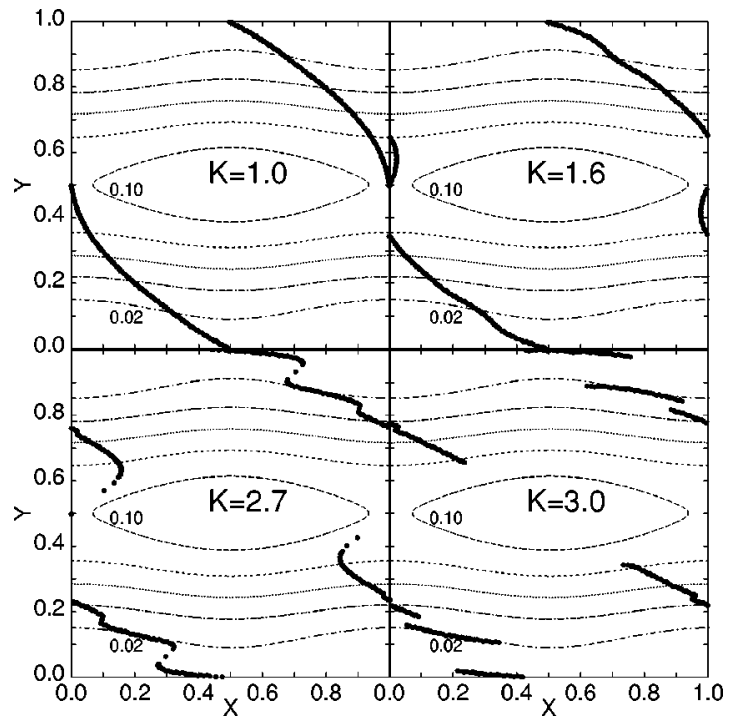

FIG. 2. Equilibrium positions folded into the 2D unit cell of $\widetilde{V}_{Q P}$ for case I with $N=233, l=144 / 233, \tau=89 / 144 \approx \tau_{g}$, and $\sigma$ $=0.25$. We show also the contour lines of $\widetilde{V}_{Q P} / K$. The different values of $K$ are indicated in each panel.

In Fig. 1 we show also the largest discontinuity of the hull function normalized to the maximum difference between the displacements of the atoms. For the FK model the hull function becomes discontinuous at the Aubry transition. For the QP case I, where we can still define a 1D periodic hull function (the structure is of rank two), the discontinuity appears at $K_{c}^{\prime} \approx 2.8$. We call this value $K_{c}^{\prime}$ because, as we will show in the following, we believe that this transition is not the analogue of that taking place at $K_{c}$ in FK and case II.

For case II one has to go over to a $2 \mathrm{D}$ hull periodic function. The size of the largest discontinuity is more difficult to define in this case and therefore we only give the value at which this 2D function becomes discontinuous; within our

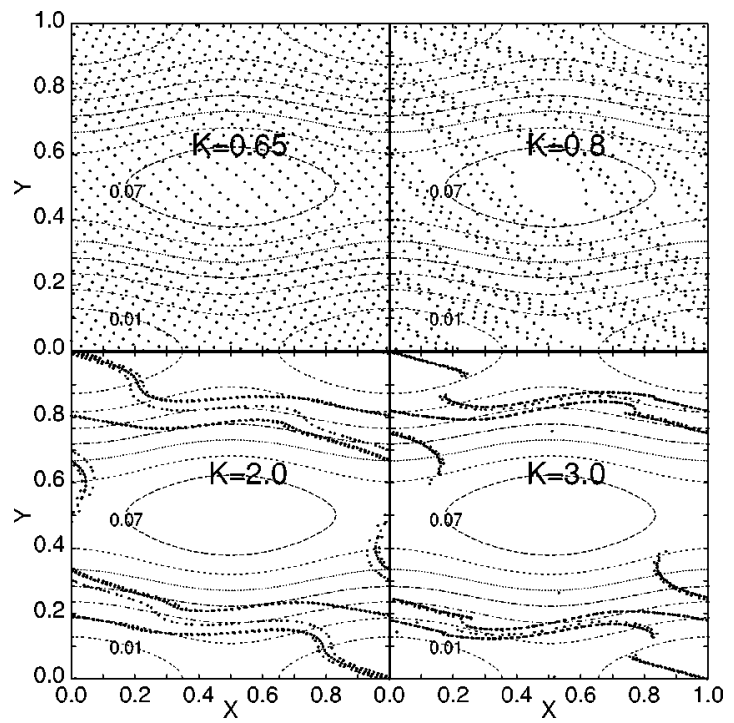

FIG. 3. Same as Fig. 2 for quasiperiodic case II with $N$ $=816, l=616 / 816, \tau=465 / 616 \approx \tau_{s}$, and $\sigma=0.25$. A larger approximant has been chosen to make the uniform distribution of points more evident. The different values of $K$ are indicated in each panel. 


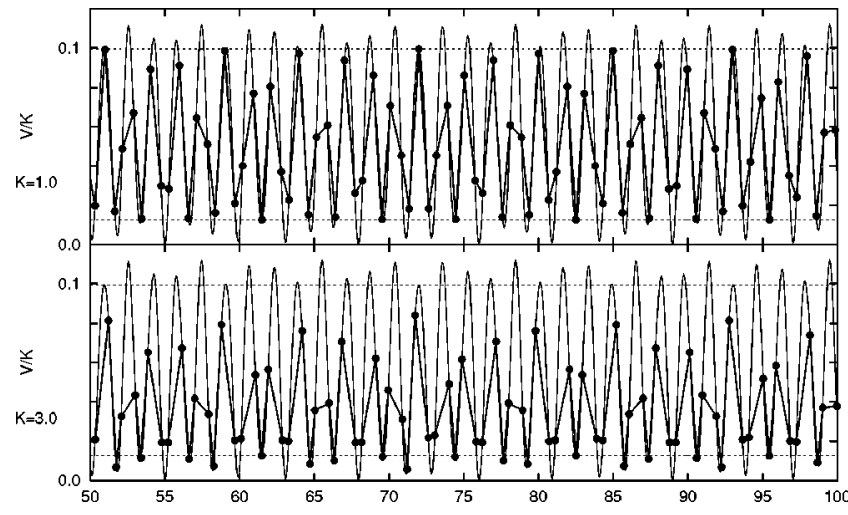

FIG. 4. Positions of particles in the potential for case I and the same values as in Fig. 1. For clarity only a portion of the chain is shown. Top panel: $K=1.0$. Notice that only potential energies between the values $2 \sigma K /(2 \pi)^{2}$ and $2(1-\sigma) K /(2 \pi \tau)^{2}$ which are given as dotted lines are occupied (see text). Bottom panel: $K$ $=3.0>K_{c}^{\prime}$. Notice that no maxima are occupied.

numerical approach we place this transition at the same value $K_{c}=0.7$ where the phonon gap opens up. The discontinuities in this function are related, as in the FK model, to the appearance of forbidden regions around maxima of the potential.

For case I, however, the pinning of the chain for any $K$ $\neq 0$ means that some regions of the potential are always forbidden if $\sigma \neq 0$ or 1 . This is why we believe that the value $K_{c}^{\prime}$ at which the hull function becomes discontinuous indicates another type of transition, which is illustrated in Fig. 4. We show two situations, one below and one above $K_{c}^{\prime}$. It is clear that already for $K<K_{c}^{\prime}$, i.e., before the occurrence of discontinuities, not all potential values are occupied but only those with energy bounded by the dotted lines. This is just another manifestation of the pinning of the chain in case I. The equilibrium positions for $K \rightarrow 0$ folded into the 2D periodic unit cell fall onto the line $Y=1 / 2-X$ implying that only the values of the potential $\widetilde{V}(X, 1 / 2-X)$ are probed. These values are bounded between $2 \sigma K /(2 \pi)^{2}$ and $2(1-\sigma) K /(2 \pi \tau)^{2}$ which are given in Fig. 4 as dotted lines. The value $2(1-\sigma) K /(2 \pi \tau)^{2}$ corresponds to the lowest local maximum of $V^{Q P}(x)$ and the appearance of discontinuities in the hull functions is related to the fact that for $K>K_{c}^{\prime}$ this energy level becomes forbidden, which means that all maxima are abandoned. In Fig. 4 for $K=3.0>K_{c}^{\prime}$ indeed no particles occupy maxima of the potential.

For case II the situation is more complex and the appearance of forbidden regions occurs in two steps, as shown in Fig. 5: At $K_{c}$ the highest maxima are abandoned and only at a much higher value $K_{c}^{\prime} \gtrsim 2.7$ all maxima are abandoned. This provides evidence that, at $K_{c}^{\prime}$, a second type of transition, similar to that occurring in case I, takes place. Two other aspects corroborate this statement: (i) at $K_{c}^{\prime}$ the phonon gap starts increasing steadily for both cases I and II (see middle and bottom panels of Fig. 1) and (ii) the equilibrium positions folded in the 2D unit cell of $V^{Q P}$ in Fig. 3 for $K$ $>K_{c}$ form lines which break up in segments at $K_{c}^{\prime}$ in a similar way as the line in Fig. 2 for case I.

On this basis, we suggest that two transitions take place for increasing potential strength, the first at $K_{c}$, due to the

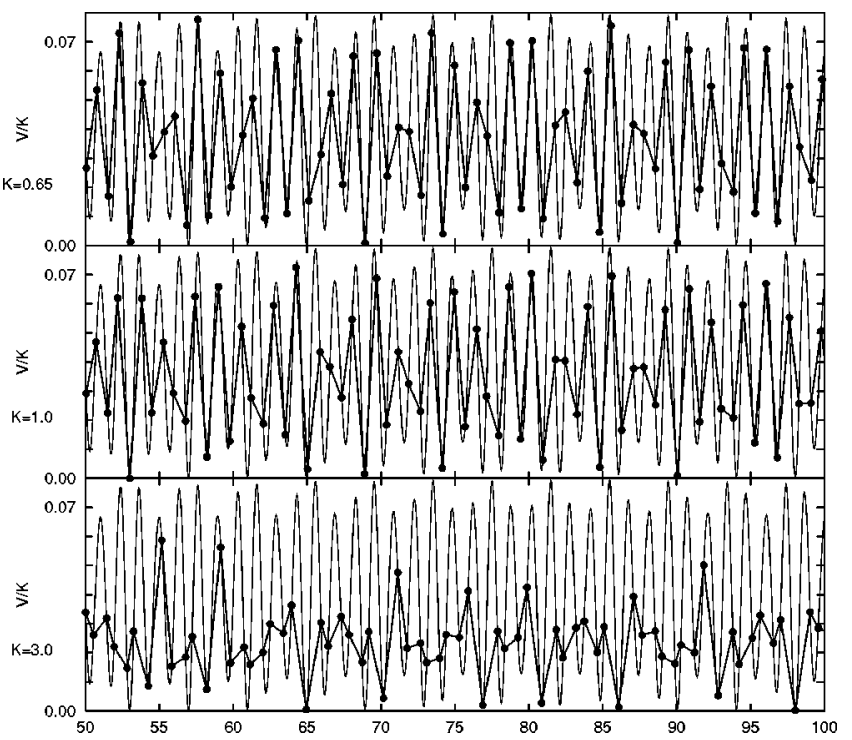

FIG. 5. Positions of particles in the potential for case II and the same values as in Fig. 1. For clarity only a portion of the chain is shown. Top panel: $K=0.65 \leqslant K_{c}$. Middle panel: $K=1.0\left(K_{c}<K\right.$ $<K_{c}^{\prime}$ ). Note that higher maxima are not occupied anymore. Bottom panel: $K=3.0>K_{c}^{\prime}$. Note that no maxima are occupied.

first appearance of forbidden regions and leading to the opening of a phonon gap, the second at $K_{c}^{\prime}$ when all local maxima become forbidden and $\omega^{2}(K)$ starts increasing monotonously. These two transitions coincide in the FK model where all maxima are at the same height. In the QP case II we observe both. For the QP case I, the first transition is absent because $\omega^{2}$ is nonzero for any $K \neq 0$ and the highest maxima are never occupied so that only the second transition takes place.

\section{B. Phonon localization}

Last, we come to the problem of localization. In Fig. 1 we have reported also the PR as defined in formula (22). In the QP cases I and II it drops steeply to zero but it is interesting that a strong tendency to localization occurs also in the FK model just around $K_{c} .{ }^{21}$ In case I, localization occurs around the first inflection point of the phonon gap, in case II it occurs at $K_{c}$. In Figs. 6 and 7 we show typical eigenvectors of the lowest phonon frequency around the transition to localization for case I and II, respectively. For comparison, in Fig. 8 , we show also those of the FK model around the Aubry transition. In all cases, the eigenvector for $K$ below localization for cases I and II, and below the Aubry transition for FK, can be qualitatively described by a modulation around the constant value $1 / \sqrt{N}$ which would describe a uniform displacement. For the FK model this description roughly applies also at, and above, the Aubry transition, but at the Aubry transition the deviations are the largest and the displacement is partly localized on a number of sites in the structure. The resemblance with the results for case I just below the localization transition is remarkable. However, while in the FK model the tendency to localization is frustrated above the Aubry transition, in case I the eigenvector of the lowest frequency mode becomes progressively more localized with exponential shape above $K \approx 1.5$, where the PR 


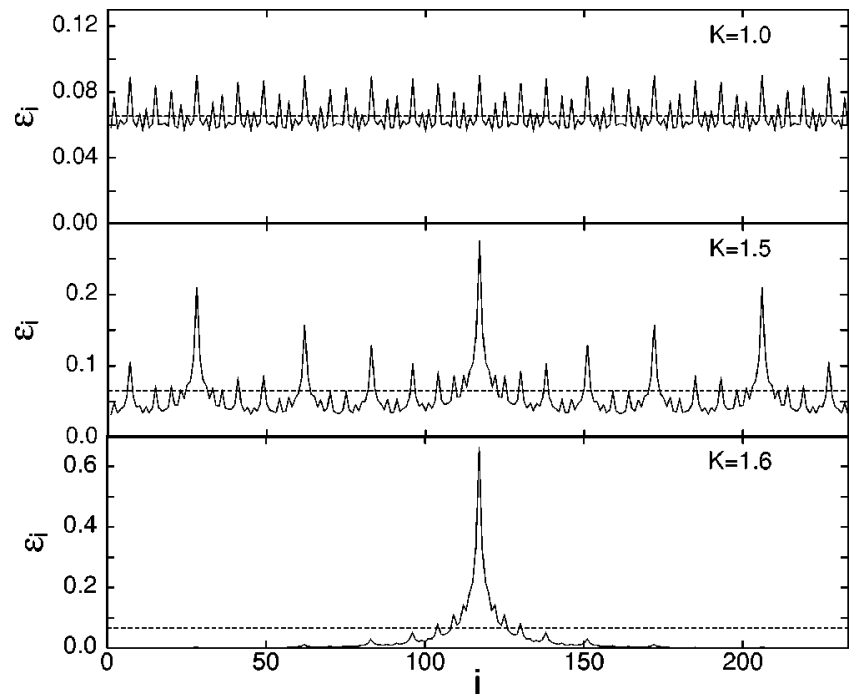

FIG. 6. Normalized eigenvector of the lowest phonon mode for the QP case I with parameters as in Fig. 1 for three values of $K$ around the appearance of localization. From top to bottom, $K$ $=1.0,1.5,1.6$. The horizontal dashed lines as in Fig. 8 .

tends to zero. Localization is very sudden and pronounced also for case II but with a more irregular pattern than for case I.

We cannot as yet provide a precise relationship relating the values of $K$ at which localization occurs with that at which the other structural transitions take place beyond the values we get from our numerical results. However we can put forward some speculations as to why localization occurs in QP potentials and it is destroyed in the FK model. We believe that an important role is played by the fact that the particles of the chain experience very different values of the potential. In periodic systems, the Bloch theorem forbids localized states, and localization in crystals is therefore always due to impurities or surfaces. For small $K$ the QP potential is a small perturbation and therefore the modes are extended

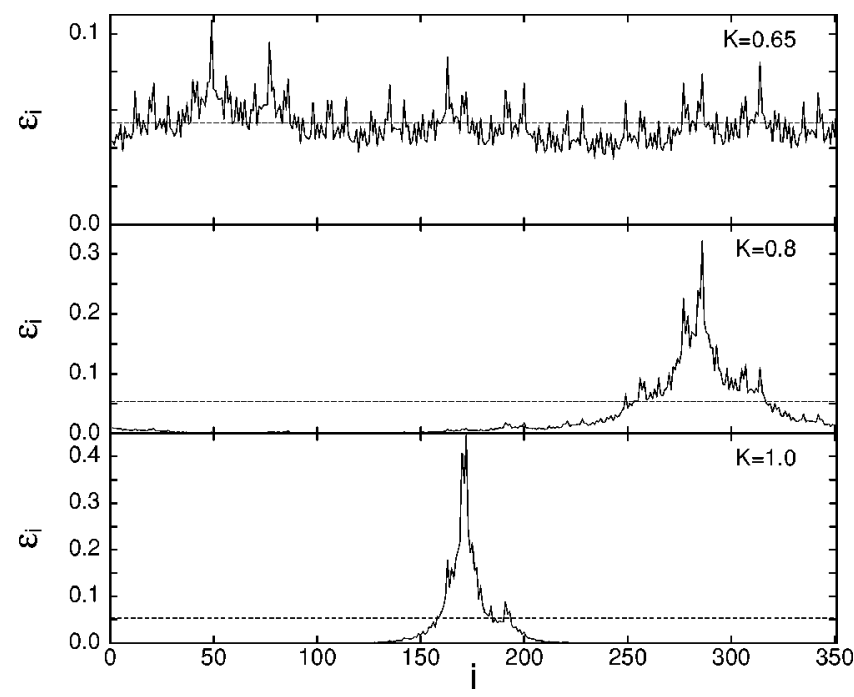

FIG. 7. Normalized eigenvector of the lowest phonon mode for the QP case II with parameters as in Fig. 1 for three values of $K$ around the appearance of localization, from top to bottom, $K$ $=0.65,0.8,1.0$. The horizontal dashed lines as in Fig. 8 .

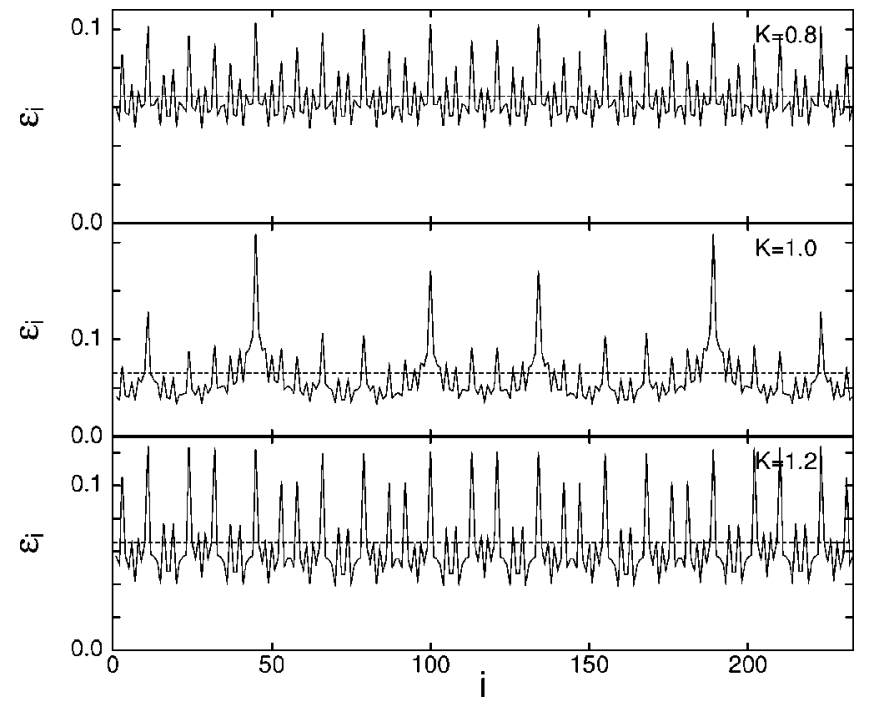

FIG. 8. Normalized eigenvector of the lowest phonon mode for the FK model with parameters as in Fig. 1 for three values of $K$ around the Aubry transition. From top to bottom, $K=0.8,1.0,1.2$. The horizontal dashed lines indicate the value $1 / \sqrt{(} N)$ which would correspond to a uniform mode.

for small $K$. Before the appearance of forbidden regions around the maxima of the potential, for increasing values of $K$, more and more particles approach the bottom of the potential wells so that the particles still occupying the maxima will acquire the character of an impurity. Indeed we find that the eigenvector is always localized on particles located at potential maxima (softest places because the second derivative of the potential is negative and has the highest absolute value). If the corresponding frequency is zero, as in FK and in case II, it is easy to displace this particle without moving all the others. It is therefore logical that at this point a transition to a new ground-state configuration, where the "impurity" particles no longer occupy the maxima of the potential, takes place. In other words, we can see localization as a precursor of the structural transition.

In turn, the new ground-state configuration can cancel the localization as it happens in the FK model. Due to the Aubry transition, there are no atoms at energy very different from all the others which can play the role of an impurity. In case II the picture is slightly more complicated because at $K_{c}$ the highest maximum is abandoned, but the localization is taken over by an atom at the top of a lower maximum, which will fall down at its turn for increasing $K$, so that, as shown in Fig. 7, the mode remains localized albeit at another site. This process also gives rise to a complex behavior of $\omega^{2}(K)$ up to the second transition where all maxima become forbidden. This transition does not necessarily cancel the localization, because due to the more complex potential the energy differences are conserved. In case I, the localized mode corresponds to a phonon with nonzero frequency so that the amount of energy required to displace particles away from the maxima is too high to cause a structural transition. As shown in Fig. 1, localization causes first an inflection and then a decrease of $\omega^{2}$, and when the phonon gap is low enough the second transition at $K \approx 2.8$ takes place. Also in this case, as in case II, this transition does not necessarily cancel the localization. 


\section{SUMMARY AND CONCLUSIONS}

In summary we have shown that the extension of the FK model to quasiperiodic potentials gives a much richer scenario as compared to the FK model. Within our model we have identified two classes of systems according to the rank of their Fourier module (cases I and II) which either display a zero frequency sliding mode or are pinned for any nonzero strength of the additional harmonic which makes the potential quasiperiodic. We have provided a criterion for the existence of a sliding mode which applies to all cases studied, including the limiting case represented by the FK model. Based on our numerical results, we propose that two possible structural transitions can take place as a function of the potential strength when the height of the potential maxima is space dependent as it is in our QP potential: The first transition signals the appearance of forbidden regions around the highest maxima and coincides with the disappearance of the sliding mode, and the second transition occurs when all maxima are abandoned and $\omega^{2}(K)$ starts growing monotonously. These two transitions coincide in the FK model where all potential maxima are at the same height. Case I presents only the second transition, case II both of them. Besides, we have studied the localization of phonons and given a rationale of why it occurs in the QP case and is suppressed in the FK model. According to our interpretation of the numerical results, localization can be seen as a precursor of the Aubry-type structural transitions. Beside the intrinsic interest, we hope to have shown that the study of quasiperiodic systems can also help in understanding the properties of simpler models like the FK.

\section{ACKNOWLEDGMENT}

One of us (T.S.E.) wishes to thank Femke Peeters for continuing support and inspiration.
${ }^{1}$ J. Frenkel and T. Kontorova, Zh. Eksp. Teor. Fiz. 13, 1 (1938); F. C. Frank and J. H. van der Merwe, Proc. R. Soc. London, Ser. A 198, 205 (1949).

${ }^{2}$ S. Aubry, in Solitons and Condensed Matter Physics, edited by A. R. Bishop and T. Schneider (Springer, Berlin, 1981), pp. 264277.

${ }^{3}$ M. Peyrard and S. Aubry, J. Phys. C 16, 1593 (1983).

${ }^{4}$ S. Aubry and G. André, Ann. Isr. Phys. Soc. 3, 133 (1980); reprinted in The Physics of Quasi-Crystals, edited by P. J. Steinhardt and S. Oslund (World Scientific, Singapore, 1987), pp. 554-595.

${ }^{5}$ J. B. Sokoloff, J. E. Sacco, and J. F. Weisz, Phys. Rev. Lett. 22, 1561 (1978).

${ }^{6}$ L. M. Floria and J. J. Mazo, Adv. Phys. 45, 505 (1996).

${ }^{7}$ O. M. Braun, T. Dauxios, M. V. Paliy, and M. V. Peyrard, Phys. Rev. Lett. 78, 1295 (1997); Phys. Rev. E 55, 3598 (1997).

${ }^{8}$ M. El-Bataouny, S. Burdick, K. M. Martini, and P. Stancioff, Phys. Rev. Lett. 58, 2762 (1987).

${ }^{9}$ S. Aubry, J. Phys. (Paris) 44, 147 (1983).

${ }^{10}$ G. Theodorou and T. M. Rice, Phys. Rev. B 18, 2840 (1978).

${ }^{11}$ O. Radulescu and T. Janssen, J. Phys. A 30, 168 (1997).

${ }^{12}$ M. Hirano and K. Shinjo, Phys. Rev. B 41, 11837 (1990); K.
Shinjo and M. Hirano, Surf. Sci. 283, 473 (1993); M. Hirano, K. Shinjo, R. Kaneko, and Y. Murata, Phys. Rev. Lett. 67, 2642 (1991).

${ }^{13}$ M. Weiss and F.-J. Elmer, Phys. Rev. B 53, 7539 (1996).

${ }^{14}$ H. Matsukawa and H. Fukuyama, Phys. Rev. B 49, 17286 (1994).

${ }^{15}$ M. Hirano, K. Shinjo, R. Kaneko, and Y. Murata, Phys. Rev. Lett. 78, 1448 (1997).

${ }^{16}$ S. E. Burkov, B. E. Koltenbah, and L. W. Bruch, Phys. Rev. B 53, 14179 (1996)

${ }^{17}$ C. M. Sokoulis and E. N. Economou, Phys. Rev. Lett. 48, 1043 (1982).

${ }^{18}$ S.-H. Kim and S. Ostlund, Phys. Rev. A 34, 3426 (1986). In this paper the definition of the spiral mean corresponds to our $\tau_{s}^{-1}$.

${ }^{19}$ The value of $K_{c}$ has been precisely estimated to be 0.971635406 for the FK model in Ref. 20, whereas our estimate of $K_{c}$ for case II and $\sigma=0.25$ is based on approximants up to 616 particles.

${ }^{20}$ R. S. MacKay, Ph.D. thesis, Princeton University, 1982. Reprinted version, in R. S. MacKay, Renormalization in Areapreserving Maps (Singapore, World Scientific, 1993).

${ }^{21}$ The participation ratio drops even more in the limiting FK model resulting from case II with $\sigma=0,1$. 